
cultures

Les cahiers de l'Acedle

9-1 | 2012

Varia : plurilinguisme et transdisciplinarité

\title{
Vous récitiez ? Eh bien, slamez maintenant!
}

\section{Camille Vorger}

\section{OpenEdition}

Journals

Édition électronique

URL : http://journals.openedition.org/rdlc/2489

DOI : $10.4000 /$ rdlc. 2489

ISSN : $1958-5772$

Éditeur

ACEDLE

Édition imprimée

Date de publication : 1 avril 2012

Référence électronique

Camille Vorger, « Vous récitiez ? Eh bien, slamez maintenant! », Recherches en didactique des langues et des cultures [En ligne], 9-1 | 2012, mis en ligne le 01 avril 2012, consulté le 16 mars 2020. URL : http:// journals.openedition.org/rdlc/2489; DOI : https://doi.org/10.4000/rdlc.2489

\section{(c) (i) (2)}

Recherches en didactique des langues et des cultures is licensed under a Creative Commons AttributionNonCommercial-NoDerivatives 4.0 International License 
"Je n'ai pas beaucoup fait l'école, les profs m'appelaient espèce d'idiot.

Si tu les croises, dis-leur que je gagne ma vie à la sueur de mon stylo." (SD) ${ }^{1}$

\section{Vous récitiez ? Eh bien, slamez maintenant !}

\section{Vers une approche ludique et créative de la poésie à l'école}

\section{Vorger Camille}

Université Stendhal Grenoble 3, Laboratoire LIDILEM

\section{Résumé}

Les textes de slam sont souvent porteurs d'un message d'invitation à l'expression, qui va bien au-delà de la pratique de la récitation, d'où le titre choisi pour cet article. Dès lors, la pratique du slam apparaît comme lieu possible d'une réconciliation avec la langue - voire avec l'école - qui passe par l'évolution des représentations vis-à-vis de celles-ci. Elle peut favoriser l'appropriation linguistique en influant sur le rapport à la langue, en jouant sur sa dimension ludique et sur les frontières entre écrit et oral, s'agissant d'écrire pour dire. Tribune d'expression collective, forum de la cité moderne, le slam est aussi un lieu privilégié d'expression individuelle et de quête identitaire. Force est de constater la qualité d'écoute lors des scènes dites "ouvertes" : nous en interrogerons les transpositions dans le contexte scolaire. Nous présenterons enfin un exemple de dispositif en CLIN ${ }^{2}$, dont les documents proposés en annexe offriront un aperçu.

\section{Mots clés}

slam, poésie, créativité lexicale, atelier d'écriture

\footnotetext{
${ }^{1}$ Souleymane DIAMANKA, "Art ignare", L'Hiver Peul, 2007. Nous utiliserons les sigles suivants pour nous référer aux corpus : GCM (Grand Corps Malade), SD (Souleymane Diamanka), AAM (Abd al Malik), M (Marco DSL), MP (Mots Paumés), R (Rouda).

${ }^{2}$ CLasse d'INitiation au français accueillant des élèves nouvellement arrivés en France, âgés de 6 à 12 ans.
} 


\begin{abstract}
Slam is both a modern forum for group expression, and a way for individuals to express themselves. As such, it reflects a double quest, for both identity and poetry: language and identity are closely linked. Moreover, slam texts are used to encourage people (auditors) to express themselves. As such, the slam experience reconciles some pupils with language, influencing the way they represent and relate to it, mixing poetry and play with words, spoken and written orders. During slam sessions, the quality of listening is exceptional; we have therefore envisaged the organization of this kind of poetic performance at school. We propose, as an example, a pedagogical approach in the specific context of a class for the integration of pupils newly arrived in France (CLIN).
\end{abstract}

\title{
Keywords
}

slam poetry, lexical creativity, writing workshop, expression

\section{Introduction}

Cet article part du constat que la poésie est - ou a été - trop souvent vécue à l'école comme une injonction, sur un mode impératif et/ou sous des modalités peu variées : l'apprentissage "par cœur", suivi de la sempiternelle récitation. Pour beaucoup, il s'ensuit un Chagrin d'école, alors que ce type d'activités pourrait permettre d'accéder précisément au cour de la langue :

Il va adorer ça, faites-lui confiance, le goût de ces mots dans sa bouche, les fusées éclairantes de ces pensées dans sa tête, et découvrir les capacités prodigieuses de sa mémoire, son infinie souplesse, cette caisse de résonance, ce volume inouï où faire chanter les plus belles phrases, sonner les idées les plus claires, il va en raffoler de cette natation sublinguistique lorsqu'il aura découvert la grotte insatiable de sa mémoire, il adorera plonger dans la langue, y pêcher les textes en profondeur, et tout au long de sa vie les savoir là, constitutifs de son être, pouvoir se les réciter à l'improviste, se les dire à lui-même pour la saveur des mots. (Pennac, 2007 : 156-157)

Or de nombreux slameurs se font les tribuns de ces désenchantés d'une approche traditionnelle de la poésie, apprise par cœur - fût-ce à contrecour - et appréhendée comme 
un lieu de contraintes. À commencer par un Grand Corps Malade qui, relatant sa "Rencontre" plutôt décevante avec la poésie à l'école, lui trouve "un air bien prétentieux", et même "vraiment relou". A contrario, sa découverte du slam s'annonce sous de bien meilleurs auspices :

Mais la poésie a insisté, elle m'a rattrapé sous d'autres formes

J'ai compris qu'elle était cool et qu'on pouvait braver ses normes ${ }^{3}$

Si cette question de la norme peut générer un blocage, l'envie d'écrire, de dire, d'écrire pour dire, devient alors le maître-mot :

Nous ne sommes pas bons élèves mais l'envie nous enivre.

Le slam serait-il une clé des champs permettant d'explorer de nouveaux espaces poétiques, à l'instar de ce cancre devenu adulte échappé du poème de Prévert?

Nous sommes le cancre au fond d'la classe

Echappé du poème de Prévert, mais nous avons grandi :

Nous disons oui avec les yeux, nous disons non avec le cæur

Et sans craie ni tableau, sous le dédain cireux des adultes prodiges

Nous crachons à la face de ce faux bonheur ${ }^{5}$

En quoi l'intervention de slameurs - en tant que poètes vivants incarnant une forme de parole libre - peut-elle renouveler l'approche de la poésie à l'école ? Dans quelle mesure l'expérience d'un atelier slam peut-elle contribuer, dans la lignée des ateliers d'écriture, à libérer l'expression en influant positivement sur le rapport à la langue et à la poésie ?

\footnotetext{
${ }^{3}$ GCM, "Rencontres", in Midi 20.

${ }^{4}$ GCM, "Le jour se lève", in Midi 20.

${ }^{5}$ Damien NOURY, "Erythèmes impudiques", in Slam entre les mots (2007 : 107).
} 


\section{Le slam, genre expressif et créatif : éléments de définition et points d'ancrage}

\section{1. À la croisée des genres, à la frontière des codes et des langues}

Forme poétique émergente, le slam se caractérise par son ouverture et son refus de se laisser "dé-finir", enfermer dans des règles strictes et formelles. De fait, il se construit à la croisée des lettres et de la musique, à la confluence des genres lyrique, épique et dramatique. Si certains slams se distinguent par une forme - et une force - éminemment poétique du fait de leur structure ou des fonctions dévolues au langage : fonctions poétique et expressive, mais aussi ludique, fonction que Pruvost et Sablayrolles (2003 : 86) proposent d'ajouter. D'autres sont ancrés dans le discours, adressés comme dans la culture hip-hop (Bazin, 1997), voire théâtralisés lors de la mise en voix qui constitue leur acte de naissance. Quelques slams enfin semblent relever du conte, tels "Le conte des 1001 peines" (R, 2007) ou du genre épistolaire comme "Lettre à mon père" (AAM, 2004).

De fait, le slam se construit à la frontière des codes oral et écrit. Il résulte en effet d'un double transcodage : de l'oral à l'écrit lors de sa conception, de l'écrit à l'oral lors de sa déclamation. "J'écris à l'oral" slame Grand Corps Malade (2008), témoignant par là-même de ce brouillage des frontières. Souleymane Diamanka, slameur sénégalais cité en exergue, parle d'oralittérature : ce mot-valise fait écho à Claude Hagège qui a proposé le terme d'orature construit par analogie avec le mot écriture, reconnaissant ainsi que "le style oral est un véritable genre littéraire" alors même que la littérature est souvent conçue "à l'exclusion de la tradition orale" (1987 : 110). Or les textes de slam sont souvent porteurs de marques propres à ces deux codes - marques d'oralité et jeux typographiques - auxquelles s'ajoute la mimogestualité lors de la déclamation: textes à géométrie variable, dont la géographie et la configuration peuvent évoluer d'une scène à l'autre. En ce sens, le slam a quelque chose de warholien : chaque texte slamé est à la fois unique et reproductible à l'infini, en changeant de lieu, de paysage ou de couleur musicale, de tonalité ou de gestuelle...

Ces slams sont donc sujets à des variations multiples, qui sont non seulement pragmatiques mais aussi inter- et intralinguales : d'une part, la présence d'alternances codiques en langues 
étrangères (code switching) traduit l'ouverture au plurilinguisme, impliquant notamment les langues d'origine de certains slameurs; d'autre part, la présence de variations diatopiques ou diastratiques - notamment argotiques - introduit la diversité au cœur même de la langue. Langue et identité se trouvent alors étroitement entrelacées (Billiez, 1996). En tant que tel, oscillant entre norme et contre-norme, le slam - et sa découverte à l'école - apparaît comme un contexte favorable à l'éclosion de la créativité lexicale et à l'expression d'une identité complexe.

\subsection{Un genre discursif situationnel, fondamentalement expressif}

Si l'on part du constat d'un renouvellement constant des genres, le slam se définit comme un genre de discours, au sens de Patrick Charaudeau (2002 : 280). En tant que tel, il se construit précisément au point d'articulation entre "les contraintes situationnelles déterminées par le contrat global de communication", "les contraintes de l'organisation discursive" et "les caractéristiques des formes textuelles." En effet, les contraintes situationnelles sont inhérentes au contexte de l'oralisation d'un texte qui a été produit dans cette perspective, à savoir une scène slam ou slam session. En oralisant son propre texte, en l'animant (Goffman $1981: 144^{6}$ ), le slameur lui donne vie et cet acte - en tant que performance - est en luimême fondateur et créatif. La scène représente donc l'enjeu réel du texte, celui-ci étant à la fois expression du "je" et "jeu" communicatif tourné vers l'autre. Quant aux récepteurs qui constituent l'audience d'une scène slam, ils sont liés par un contrat de communication fondé sur l'écoute et l'échange: "un moment d'écoute, un moment de tolérance, un moment de rencontres, un moment de partage" selon la définition de Grand Corps Malade (document 1).

$\mathrm{Au}$ demeurant, la frontière entre scène et public - soit le quatrième mur au théâtre (Diderot, 1758)-est floue, de sorte que les slameurs deviennent potentiellement auditeurs et inversement, selon le principe de la scène ouverte. Il en résulte une qualité d'écoute remarquable lors des scènes slam, au cours desquelles les participants - qu'ils soient slameurs ou slamophiles - peuvent être amenés à réagir ou à interagir. Genre discursif voire

\footnotetext{
${ }^{6}$ "In short, he is the talking machine, a body engaged in acoustic activity, or, if you will, an individual active in the role of utterance production. He is functioning as an 'animator'."
} 
interdiscursif - les interpellations du public ou encore d'un slameur à un autre étant fréquentes comme dans le rap-, le slam est fondamentalement expressif, porteur d'une multimodalité qui lui est consubstantielle : mimogestualité, intensité et intonation des voix, expressivité de la substance sonore, des mots qui claquent ${ }^{7}$, des corps et des visages...

Les origines du slam et le champ sémantique du $\operatorname{mot}^{8}$ lui-même renvoient d'ailleurs à cette expressivité fondamentale, et au-delà, à l'arme des mots, brandis comme autant de coups de poings lors de tournois poétiques ${ }^{9}$. Image que l'on retrouvera au détour de certains flyers, voire de certains textes : "Slam, pour toutes ces portes claquées au nez, Slam, pour tous ces plongeons dans la foule, Slam ! "10

\subsection{Expressivité et réflexivité : une langue-miroir}

Aussi le slam est-il à la fois écriture du je, jeu poético-identitaire et expression du jeu dans la langue : un jeu fondé sur la variété et l'expressivité des mots, des voix, des corps... Si Jean Pierre Goudaillier évoque un fonctionnement "en miroir" (1997 : 32) pour décrire certains aspects du Français Contemporain, nous proposons ici le concept de langue-miroir pour rendre compte du mimétisme et de la réflexivité qui caractérisent le slam. A l'instar des auteurs romantiques qui nous donnent à lire les paysages comme autant de miroirs de leurs états d'âme, c'est bien une langue-miroir que les slameurs nous livrent à travers leurs textes. Celle-ci se fait le reflet d'une identité individuelle plurielle (avec des appartenances multiples), d'une identité collective (avec l'appartenance éventuelle à un collectif de slameurs), d'une identité artistique en construction (celle du mouvement slam) et d'une identité stylistique (artistique) propre à chaque slameur. Miroir d'une quête, d'un questionnement, d'un mouvement ou d'une mouvance, cette langue que les slameurs nous

\footnotetext{
${ }^{7}$ Tel est le sens premier du verbe to slam, "claquer".

${ }^{8}$ Si l'on se réfère au dictionnaire Longman : 1. DOOR/GATE : "If a door, gate etc slams, or if someone slams it shuts with a loud noise" 2. PUT STH SOMEWHERE : "to put something on or against a surface with a fast violent movement" 3. Slam on the brakes : "to make a car stop very suddenly" 4. CRITICIZE STH : "a word used especially in newspapers" (nous soulignons les traits sémiques qui nous paraissent importants).

${ }^{9}$ C'est une approche possible du slam à l'école, même si ce n'est pas celle que nous avons choisie de développer. Voir à ce sujet l'ouvrage suivant : Duval, C. et alii (2008). 20 ateliers de slam poésie. Retz.

${ }^{10}$ Antoine Faure/Tô, (2007). "Slam" . In Le slam, poésie urbaine. Mango.
} 
donnent à entendre et à voir en la jouant sur scène apparaît fondamentalement réflexive en tant que porteuse d'une réflexion sur le slam en particulier et la poésie en général :

On ne peut pas être et avoir été. On ne peut pas laisser la poésie se scléroser. C'est presque notre devoir de nous affairer pour la faire évoluer. (...) Elle a besoin d'une cure de DHEA, de jouvence pour se repaître, de rencontrer d'autres êtres compulsifs face aux lettres, d'autres façons de la faire danser, d'autres dictions plus condensées. ${ }^{11}$

\section{Pour une approche renouvelée de la poésie à l'école}

En tant qu'art vivant, le slam se distingue par cette capacité à "créer une collectivité humaine, ouverte, généreuse, portée par une langue" ainsi que "le pouvoir de rendre leur efficacité aux valeurs et aux symboles véhiculés par cette langue." ${ }^{12}$ De fait, de nombreux slameurs évoquent cet enjeu au sein-même de leurs textes : "Nous serons les meilleurs élèves de l'école fraternelle. "13

\subsection{Ecrire pour dire ou la découverte de l'oralittérature}

La démarche proposée dans le guide conçu par le collectif $129 \mathrm{H}$ insiste sur la valeur non dogmatique de cette méthodologie ${ }^{14}$ :

L'idée est de présenter l'écriture comme un moyen d'expression personnelle, esthétique et ludique, qu'il faut s'approprier en respectant son identité propre. $(\mathrm{sd}: 37)$

L'expression de l'identité individuelle est donc posée comme fondement de la voie/voix dont chaque participant est en quête à travers l'écriture et l'oralité, et doit permettre d'explorer "la richesse de la langue française et de ses variantes (argot, verlan)". En d'autres termes, la langue est ici envisagée dans toute sa diversité, quitte à en explorer les marges ou le jeu. Au-delà de la "familiarisation avec la discipline", le "recours systématique au jeu" - avec un savant mélange de jeux d'écriture, de jeux d'oralité et de jeux scéniques - est valorisé,

\footnotetext{
${ }^{11}$ Marco DSL, " Vous n'êtes plus seuls", Allons à l'essentiel...

${ }^{12}$ Préface du guide cité, par Nicolas Roméas, p.6.

${ }^{13}$ BANZAÏ, J. \& DIAMANKA, S. (2007). J'écris en français dans une langue étrangère. Paris : Editions Complicités.

${ }^{14}$ Ecrire et Dire. Petit guide méthodologique pour l'animation d'ateliers Slam. Collectif 129H. Mairie de Paris/FSE.
} 
ainsi que "l'aller-retour entre l'écrit et l'oral" qui demeure une caractéristique fondamentale du slam. En effet, si "l'écriture est la matière première de l'interprétation sur scène", c'est bien cette finalité du passage face à un public, et donc du passage à l'oral, qui conditionne et motive l'expression. Cette étape cruciale est donc un passage obligé de chaque séance d'un atelier slam mais à la différence de la récitation, les élèves sont invités à déclamer leurs propres textes. C'est à ces conditions que pourra être développée "l'approche positive des mots, du langage comme outil d'expression poétique, libre des contraintes purement scolaires." (sd : 41) Cette démarche se veut dégagée des pressions inhérentes au code écrit : "Le slam se joue à l'oral et ce type d'obstacle ne doit pas dévaloriser le potentiel de chaque participant." Dès lors, le slam créé par chacun des participants pourra devenir aussi volatile et éphémère qu'un "papillon en papier" :

Même s'il est né de ma plume si tu l'as aimé et qu'il t'a plu

Ce n'est plus mon poème mais un papillon en papier (SD, 2007)

\subsection{On marche mots dans la main $(R, 2007)$ ou l'expérience de la matérialité des mots}

Cette formule imagée ("mots dans la main") illustre le procédé - récurrent dans le slam - de délexicalisation ou palimpseste verbal ${ }^{15}$ si l'on se réfère à la terminologie de Galisson (1995). D'après ce dernier, il s'agit d'"une manière de revisiter, de rajeunir les clichés" qui consiste à "transformer un groupement stable en groupement libre, à désunir par regrammaticalisation une suite de morphèmes". Autant de palimpsestes verbaux résultant du chevauchement d'un sous-énoncé lexicalisé et d'un sur-énoncé résultant de la déconstruction - ou délexicalisation - du sou-énoncé de base : en l'occurrence, "(marcher) main dans la main", ou encore "(faire) du prose à porte" (R, 2007, nous soulignons). Ils constituent une marque de connivence, un moyen pour les locuteurs de "baliser leur espace de communication". De fait, le décodage s'effectue non seulement au niveau langagier mais aussi au niveau culturel, d'où l'idée de palimpseste verbo-culturel, la compréhension reposant sur le présupposé d'une lexiculture commune. En tant que tel, le palimpseste représente une source de difficultés pour un public FLE (Français Langue Etrangère),

\footnotetext{
${ }^{15}$ Le palimpseste évoque le parchemin dont le texte initial a été effacé, puis remplacé par un autre.
} 
comme le soulignent les auteures du Vocabulaire en classe de langue (2009 : 217). Il s'agit alors de repérer la culture dans les mots en analysant "la charge culturelle partagée" (Galisson, op.cit.) et ce, à partir d'un corpus de palimpsestes issus de ressources médiatiques (titres d'articles de presses, slogans publicitaires...). Or un tel décodage lexico-culturel serait tout à fait envisageable à partir d'un corpus de slams.

Pour en revenir au palimpseste cité, notons qu'il reflète non seulement l'idée de solidarité (sous-énoncé) inhérente au slam, mais aussi le principe selon lequel les mots sont appréhendés dans leur matérialité (sur-énoncé). Il s'agit là d'un point de convergence dans les entretiens que nous avons réalisés avec des slameurs. Ainsi Rouda, que nous avons invité à s'exprimer sur le sens de cette délexicalisation, nous a-t-il expliqué que le fait de matérialiser les mots, de les rendre plus concrets aux yeux des enfants permet de "dédramatiser l'écriture" (document 2). L'exemple du "combat de rimes" ou clash auquel il fait allusion montre comment les mots sont saisis et manipulés dans leur matérialité sonore, ce qui permet de libérer l'écriture. Souleymane Diamanka, dans l'entretien consacré à Héloïse Guay de Bellissen pour son ouvrage sur le slam, explique qu'il procède selon cette même démarche: "je leur dis qu'ils sont entourés de moi, même toi, t'es un mot, ton prénom, c'est un mot" (2009: 153).

Dans cette perspective, le travail sur les prénoms - les lettres du prénom mais aussi les sonorités et autres mots cachés dans son prénom, ce qui implique les phénomènes d'homophonie - est une entrée privilégiée dans les ateliers slam. À titre d'exemple, l'animatrice-slameuse avec laquelle nous avons travaillé (voir infra et document 3) a proposé, en guise d'entrée en matière pour un atelier slam mené en classe de CM2, une séance consistant dans la recherche d'un blase. Ce mot d'argot d'origine douteuse (Rey, 2007) désigne un "faux nom" ou pseudonyme qui reste, pour un jeune de cité, la marque de son identité. Pour un slameur, il reflète son identité scénique et nous avons repéré, dans le slam comme dans le rap, une esthétique des pseudonymes (Lapassade \& Rousselot, 1998 : 91). Pour nos élèves, cette séance a été un formidable déclencheur d'écriture créative comme en témoignent leurs productions. Si l'animatrice-slameuse avait donné l'exemple de son propre blase "Boutchou", issu par troncation de son nom de famille et homophonie de l'expression lexicalisée "bout d'chou" (prononcée [butfu] par assourdissement), certains 
élèves ont réinvesti ce procédé sous forme de calembours ("Thé au lait" pour Théo). D'autres se sont intéressés à la matière visuelle des mots et ont joué avec les lettres de leurs prénoms. Il en a résulté des anagrammes (Eliott $\rightarrow$ Etoile) et autres formes de verlan graphique (Alix $\rightarrow$ Xila) faisant écho, dans les procédés utilisés, aux blases choisis par des slameurs ou rappeurs confirmés ${ }^{16}$. Une telle séance d'entrée en matière soit d'entrée en slam a amené les élèves à se construire une identité scénique qui leur permettra ensuite de s'exprimer plus librement à l'oral, face à un public.

\subsection{J'ai vu des oreilles plein leurs yeux (GCM) ou la notion d'horizon d'écoute}

Issue de la théorie de la réception de Hans Robert Jauss, la notion d'horizon d'attente est définie en ces termes :

Le texte nouveau évoque pour le lecteur (ou l'auditeur) tout un ensemble d'attentes et de règles du jeu avec lesquelles les textes antérieurs l'ont familiarisé et qui, au fil de la lecture, peuvent être modulées, corrigées, modifiées ou simplement reproduites (1978: 51).

Elle s'applique aux livres pour lesquels nous avons, en tant que lecteurs experts, construit des attentes sur les plans générique, textuel et culturel. En ce qui concerne le slam, nous proposons la notion d'horizon d'écoute pour rendre compte de la qualité d'écoute manifestée lors de sessions slam - quel qu'en soit le contexte - et d'une ouverture à tous les possibles linguistico-poétiques qui la caractérisent. De fait, tous les textes sont accueillis avec respect et bienveillance, sans a priori manifeste. Quelques "règles du jeu" (document 1) sont néanmoins imposées lors d'une scène ouverte : la brièveté (le texte ne doit pas excéder trois minutes), l'absence d'accompagnement musical (il doit être déclamé a capella) et d'accessoires. L'horizon d'écoute pourra être conditionné par le nom de scène du slameur, mais aussi par des écoutes antérieures, s'agissant de slameurs confirmés - et d'auditeurs initiés - ayant réussi à construire un univers et un style qui leur sont propres. Les transitions ménagées d'un texte à l'autre dans la succession des slameurs caractéristique d'une scène ouverte peuvent aussi influer sur l'horizon d'écoute : d'une certaine manière, le slameur ou le cas échéant l'animateur de la scène - "annonce la couleur", la tonalité de son texte. Il

\footnotetext{
${ }^{16}$ YSAE, verlan graphique de EASY pour un rappeur marseillais.
} 
met les auditeurs en condition pour entendre son slam ou le message dont il est porteur. L'environnement musical peut enfin prendre en charge cette préparation du public, en contribuant à créer une atmosphère favorable à l'écoute de tel ou tel texte. Les musiques électroniques - dites amplifiées - permettront ainsi d'introduire une thématique plus ou moins actuelle, telle que la pollution des villes dans le texte "Apnée" de Mots Paumés ${ }^{17}$. En effet, elles amplifient la tension dramatique interne à ce texte et souligne l'harmonie imitative du flow qui caractérise sa déclamation.

Lors d'un atelier slam, l'écoute représente l'une des valeurs fondamentales véhiculées (document 2) et des moyens multiples peuvent être mis en œuvre pour la favoriser, sans aller jusqu'à impliquer des élèves comme jury selon la tradition américaine. Un slameur comme le grenoblois Bastien Maupomé (alias "Mots Paumés"), qui se distingue par sa capacité d'adaptation à son auditoire, met à profit des stratégies telles que l'interpellation ou une interrogation initiale - soit une consigne d'écoute - visant à finaliser celle-là en suggérant une tâche aux auditeurs. Dans un texte sur les fromages, il demande par exemple aux enfants de compter le nombre de mots relevant de ce champ lexical : exercice-jeu qu'ils se font un plaisir d'exécuter.

\section{Vers la recherche action : un exemple d'atelier slam mené en CLIN}

\subsection{Contextualisation}

Les documents annexés à cet article rendent compte d'un atelier slam mené en CLIN avec l'intervention d'une slameuse ${ }^{18}$ : il s'agit de la troisième séance de l'atelier. Elle a fait l'objet d'un enregistrement vidéo qui a permis la réalisation d'un synopsis d'observation et la transcription de l'échange entre deux élèves placés en situation de coproduire un texte (documents 4 et 5). Cette phase de production écrite succède à des jeux d'interprétation et jeux de rythmes qui constituent la première partie de la séance : l'entrée par la musique permet une activité commune, accessible à tous quel que soit leur niveau de maîtrise de la

\footnotetext{
${ }^{17}$ http://mots-paumes.blogspot.com/ (consulté le 9/03/12)

${ }^{18}$ Katia Bouchoueva, "Boutchou" : http://www.myspace.com/boutchoukatia
} 
langue, au sein d'un groupe-classe foncièrement hétérogène. En guise de transition et de préparation à l'expression écrite, les élèves sont ensuite mis en situation d'écouter un slam de Rouda intitulé "Paris canaille", tâche dont témoigne la prise de notes de l'élève M. (document 4). A partir de cette écoute, chaque dyade est invitée à choisir une ville qui sera au cœur de son slam. Les deux élèves dont la production et l'interaction figurent en annexe ont alors demandé s'ils pouvaient inventer "une ville à deux têtes" - à l'image de cette dyade - qui plus est "remplie de nomades", ce qui n'est pas sans évoquer leur propre parcours migratoire.

\subsection{Un contexte favorisant l'expression de soi et la créativité lexicale}

Si l'on se réfère au document 4, il apparaît que le titre choisi pour ce texte - "Normat" - est une première manifestation de créativité lexicale : il s'apparente à un mot-valise - même si l'élève n'a pas réalisé à l'écrit la fusion des deux unités - qui fusionne les deux prénoms des élèves concernés (Nordin/Mathieu). Ces élèves se sont ici clairement inspirés d'un slam qui a fait l'objet d'une précédente séance et d'un travail d'interprétation en duo : il en résulte une interaction fertile entre écoute et écriture. Co-écrit et co-interprété par Souleymane Diamanka et John Banzaï, ce texte est intitulé "Soleil Jaune". Les élèves ont bien perçu le jeu de paronymie (Souleymane $\rightarrow$ soleil / John $\rightarrow$ jaune) et ont spontanément cherché à le réinvestir dans leur production. Aussi leur slam est-il construit sur des procédés d'homophonie (Mat/maths, airs/aires) et de paronymie (Nomades/Normat) qui témoignent d'un jeu sur la langue dans lequel les deux sujets se sont impliqués. En ce sens, le slam est à la fois écriture du je et écriture en jeu(x), expression et exploration du jeu - des marges - de la langue.

\subsection{Une démarche colludique ${ }^{19}$ et réflexive}

En effet, les élèves ont bien perçu la dimension ludique inhérente au slam : dès le titre de leur texte, ils ont joué sur la matière visuelle (les lettres) et sonore de leurs prénoms. C'est ici à une dimension colludique (de colludere, "jouer ensemble") qu'ils accèdent et cet enjeu nous paraît essentiel dans les ateliers slam, s'agissant de jouer ensemble avec les mots.

\footnotetext{
${ }^{19}$ Nous proposons ce concept de colludique comme synthèse de la fonction ludique et d'une fonction qui serait fortement conniventielle au sein d'un groupe donné. La dimension collective de ce jeu nous paraît essentielle.
} 
L'échange de type métalinguistique qui s'ensuit (document 5) traduit des désaccords portant sur la mise en mots (syntaxe) et la mise en forme (ponctuation) du texte. L'élève $\mathrm{M}$. manifeste des inquiétudes quant au transcodage que représente le passage à l'écrit : il s'agit d'un élève lusophone, qui plus est en difficulté à l'écrit. Pour cet élève, la prégnance du contrat didactique ralentit considérablement le processus d'écriture. L'élève N., quant à lui, se laisse porter par son inspiration, jouant au passage sur le sens propre et le sens figuré de l'expression "avoir un mot sur le bout de la langue". Cet échange apparaît révélateur d'un travail possible sur le défigement des expressions figées comme déclencheur de créativité lexicale et d'investissement affectif. La réaction finale des élèves vis-à-vis de la caméra traduit la dimension intime de cette phase d'écriture, fût-elle duelle. En tout état de cause, la situation proposée et décrite s'avère propice à une expression et à une créativité qui trouveront leur aboutissement sur la scène improvisée dans la classe. Celle-ci permettra une expressivité reposant également sur les voix et les corps (Pierra, 2006), dès lors que les élèves animeront leur slam aux sens premier et goffmanien (1981) du terme.

\subsection{Des résultats encourageants}

En donnant vie à leurs propres textes et voix à leurs propres mots, c'est autant au cœeur $d u$ slam qu'au cœur de la langue que ces élèves ont plongé. Ils ont trouvé leur voix dans cette langue seconde qui leur était étrangère quelques mois auparavant. En témoignent les entretiens que nous avons menés le 17 juin 2008, à l'issue de la séquence expérimentée. A la question, "Pour toi, le slam, qu'est-ce que c'est ?", un élève a répondu "c'est des cliques et des claques", traduisant par cette pirouette lexicale, son appropriation du sens originel du concept de slamming (to slam, "claquer") et du jeu de mot qui réside ici dans une forme de délexicalisation du phrasème "prendre ses cliques et ses claques". On retrouve l'acception première du verbe dans cette autre réponse d'une élève : le slam l'a aidée, précise-t-elle à "apprendre le français en claquant les mots". Et sa camarade d'ajouter alors, prise dans un enthousiasme colludique: "On fait claquer la langue, la poésie, les mots et même le cerveau !". Aux yeux de cette élève, le slam se distingue de la poésie par la capacité d'invention, de création qu'il éveille, là où la représentation de la poésie semble se réduire à l'exercice de récitation: "la poésie, on la dit, mais le slam, on l'invente !" Un élève conclura : "le slam, c'est une chose qui fait plaisir, la poésie c'est plus difficile..." A la 
Poésie avec un grand "p" - mot qui trop souvent effraie -, le slam répond donc par une démarche qui semble plus accessible: il s'agit là d'une entreprise de désacralisation de l'écriture poétique conforme au dessein initial de son fondateur ${ }^{20}$.

\section{Conclusion}

Porteur d'une langue plurielle, langue du "je" et du "jeu" pour les slameurs en herbe comme pour les slameurs confirmés, la découverte du slam en contexte scolaire représente un lieu privilégié d'investissement subjectif - voire de quête identitaire - et de créativité. En tant que telle, sa pratique peut favoriser l'appropriation linguistique (Pierra, 2006) - a fortiori dans le cas d'élèves nouvellement arrivés en France -, ou encore la réconciliation avec une langueculture dont ils pourraient se sentir exclus. En ouvrant aux élèves une autre voie, en les initiant à un nouveau rapport à la langue, les ateliers slam pourront alors, via une approche renouvelée et ludique de la poésie, permettre à certains de trouver leur voix ou tout au moins l'envie d'écrire :

Le jour se lève et la joie se livre, la soif se lit sur nos lèvres, tu devrais nous suivre / Si notre heure est brève, nous allons quand même la vivre

Nous ne sommes pas bons élèves mais l'envie nous enivre / Alors à ton tour ouvre les yeux, approche-toi et observe avec curiositél Le souffle et l'enthousiasme d'une brigade de poètes sortis tout droit de l'obscurité (GCM, 2006)

\footnotetext{
${ }^{20}$ Marc Smith, ex-ouvrier du bâtiment, a créé le slam à Chicago dans les années 80 avec cet enjeu de démocratisation de la parole poétique.
} 


\section{Références}

\section{Bibliographie :}

Billiez, J. (1996). "Poésie musicale urbaine : langue et identité entrelacées". In Julliard, C. \& Calvet, L.-J. (éd.). Les politiques linguistiques, mythes et réalités. Beyrouth. AUPELFUREF.

Cavalla, C., Crozier, E., Dumarest, D., Richou, C. (2009). Le vocabulaire en classe de langue. Paris : Clé international.

Charaudeau, P. \& Maingueneau, D. (2002). Dictionnaire d'analyse du discours. Paris : Seuil.

Diderot, D. (1758). Discours sur la poésie dramatique, In Oeuvres esthétiques. Ed. P. Vernière. (1968). Paris : Garnier. pp.183-287.

Galisson, R. (1995). "Les palimpsestes verbaux : des révélateurs culturels remarquables, mais peu remarqués". In Cahiers du français contemporain 2, La locution en discours. Paris : Crédif - Didier érudition.

Goffman, E. (1981). Forms of talk. Oxford : Basil Blackwell.

Goudaillier, J. P. (1997). Comment tu tchatches? Dictionnaire du Français contemporain des cités. Paris : Maisonneuve et Larose.

Guay De Bellissen, H. (2009). Au cour du slam: Grand Corps Malade et les nouveaux poètes. Monaco : Editions Alphée/Jean-Paul Bertrand.

Jauss, H. R. (1978). Pour une esthétique de la réception. Paris : Gallimard.

Lapassade, G., \& Rousselot, P. (1998). Le rap ou la fureur de dire. Paris : Ed. Loris Talmart.

Pennac, D. (2007). Chagrin d'école. Paris : Gallimard.

Pierra, G. (2006). Le corps, la voix, le texte, Arts du langage en langue étrangère. Paris : L'Harmattan. 
Pruvost, J., \& Sablayrolles, J.-F. (2003). Les néologismes. Paris : PUF.

Rey, A. (2007). Dictionnaire historique de la Langue Française. Paris : Le Robert.

Trimaille, C. (1999). De la planète Mars... Codes, langage, identités: étude sociolinguistique de textes de rap marseillais. Mémoire DEA sous la direction de J. Billiez. Grenoble : Université Stendhal, Grenoble 3.

Vorger, C. (à paraître). A la recherche de l'argot perdu : le slam et ses néostyles, Actes du colloque d'argotologie "Expressivité vs identité dans les langues", Brno, 19-21 février 2010.

Collectif 129H (2007). Écrire et dire, Petit guide méthodologique pour l'animation d'ateliers Slam (sd). Paris : Mairie de Paris.

\section{Discographie, recueils et anthologies :}

Abd Al Malik (2004). Le face à face des cours. Atmosphériques.

Abd Al Malik (2008). Dante. Universal Music.

Grand Corps Malade (2006). Midi 20. Editions Raoul Breton/Anouche Productions, Universal Music.

Grand Corps Malade (2008). Enfant de la ville. Editions Raoul Breton/Anouche productions. Universal Music.

Diamanka, S. (2007). L'Hiver Peul. Universal Music.

Diamanka, S. \& John Banzaï, J. (2007). J'écris en français dans une langue étrangère. Paris : Les éditions Complicités.

Martinez, S. (2007). Slam entre les mots : anthologie. Paris : Editions de La Table Ronde, coll. "La petite vermillon".

Mots Paumés Trio slamusic (2009). Songes déments.

Rouda (2007). Musique des lettres. Harmonia Mundi.

Faucheur, J. (2006). Le slam, poésie urbaine. Paris : Mango, coll."album Dada". 


\section{Annexes}

\section{Document 1 : Définition de GCM ${ }^{21}$}

C'est quoi le slam?

Il y a évidemment autant de définitions du slam qu'il y a de slameurs et de spectateurs des scènes slam. Pourtant il existe, paraît-il, quelques règles, quelques codes :

- les textes doivent être dits a cappella ("sinon c'est plus du slam"?)

- les textes ne doivent pas excéder 3 minutes (oui mais quand même des fois, c'est 5 minutes...)

- dans les scènes ouvertes, c'est "un texte dit = un verre offert" (sauf quand le patron du bar n'est pas d'accord...)

Bref, loin de ces incertaines certitudes, le slam c'est avant tout une bouche qui donne et des oreilles qui prennent. C'est le moyen le plus facile de partager un texte, donc de partager des émotions et l'envie de jouer avec des mots.

Le slam est peut-être un art, le slam est peut-être un mouvement, le slam est sûrement un Moment... Un moment d'écoute, un moment de tolérance, un moment de rencontres, un moment de partage.

\section{Document 2 : Extrait de l'entretien avec le slameur Rouda}

Date et lieu de l'entretien : le 27/10/08 à Montreuil.

Les locuteurs sont désignés par leurs initiales: C. (Camille, enquêtrice), R. (Rouda, enquêté), L. (Lyor, membre du collectif 129H).

C: Est-ce que tu pourrais expliciter cette image: "On marche mots dans la main"? Comment tu l'as trouvée et que signifie-t-elle pour toi ?

$\mathrm{R}$ : Ben, là, j'écrivais un texte sur la langue et l'usage de la langue et voilà... J'ai détourné l'expression "main dans la main", et ça m'est venu comme ça, quoi !

\footnotetext{
${ }^{21}$ Voir sur le site officiel de GCM : http://www.grandcorpsmalade.com/slam.htm
} 
$\mathrm{C}:$ L'image renvoie aussi à une matérialité des mots, de la langue ?

$\mathrm{R}$ : Ouais... Parce que par exemple un truc qu'on dit beaucoup aux gamins dans les ateliers d'écriture, notamment ceux qui ont des difficultés de vocabulaire, leur dire que tout ce qui nous entoure est un mot.

C : Rendre les mots plus concrets.

$\mathrm{R}$ : Les matérialiser, mais ce sont des mots : le mot table, le mot téléphone, cartable, étui... Les mots sont palpables autour de nous.

$\mathrm{C}:$ Ça permet aussi de dédramatiser l'écriture de partir du concret comme ça.

$\mathrm{R}$ : Notamment dans la recherche de rimes. Les gamins ont du mal, quand ils sont petits, à capter le concept de rime. Donc souvent on leur fait ça, on va dire, je te donne un mot on va faire un combat de rimes, un clash de rimes, je te donne le mot "voiture", tu vas me dire "ceinture", "peinture", "rature"... Je leur dis "Regarde autour de toi, détache-toi et regarde les mots qui sont autour de toi : mur, chaussure" (...)

$\mathrm{C}$ : Est-ce qu'il y a un âge qui vous paraît particulièrement favorable à ces ateliers, à la fois pour l'écriture et pour l'oralisation?

L : Ça s'inverse avec le temps en fait. C'est-à-dire que quand ils sont plus jeunes, ils vont avoir plus de facilités à aller sur scène mais pas grand-chose à raconter, ou alors pas d'idées...

C : L'écriture est plus difficile mais ils sont moins inhibés.

L : Et puis les ados, ils vont avoir plein de choses à dire, des facilités à se livrer sur le papier, mais dès qu'il va s'agir d'aller devant un public, ça va être un peu plus compliqué. Ça se rééquilibre après, mais ça dépend aussi des caractères, des parcours... (...)

$\mathrm{C}$ : Alors, il est aussi question de valeurs: quelles sont les valeurs que vous souhaitez transmettre?

R : Essentiellement l'écoute. Même nous, dans notre travail quotidien, on essaie de s'imposer ça! D’ailleurs, où est le bâton de parole ? (rires)

$\mathrm{N}$ : Justement, je l'ai pas retrouvé... Je pense que le mec, il l'a gardé pour garder la parole ! (rires) 
L : L'écoute, oui, et puis, je sais pas si c'est une valeur mais ce qu'on essaie de montrer à travers ces ateliers, c'est qu'on peut se servir de la langue autrement que d'une manière très scolaire et formelle par nécessité. C'est-à-dire que souvent, les gamins considèrent que s'exprimer bien, c'est presque un devoir, au sens scolaire, et du coup, on leur montre à travers les ateliers qu'ils peuvent utiliser ça pour exprimer vraiment ce qu'ils ressentent, et aussi faire passer ce message à des gens à qui ils n'auraient pas l'occasion de parler dans leur vie quotidienne. Adapter leur niveau de langage aux gens...

\section{Document 3 : Synthèse des choix de blases par des élèves de $\mathrm{CM}^{22}$}

\begin{tabular}{|l|l|l|}
\hline Prénom (Nom) & Blase & Procédé \\
\hline Alix & Xila le minibras & $\begin{array}{l}\text { Verlan graphique + périphrase } \\
\text { intégrant une assonance }\end{array}$ \\
\hline Amir & Prince Rima & Verlan graphique + trad. de l'arabe \\
\hline Axel & Axelix & $\begin{array}{l}\text { Suffixation (par analogie avec } \\
\text { «Alix », «Astérix ») }\end{array}$ \\
\hline Eliott & L'étoile & Anagramme \\
\hline Emma & Mama & Redoublement hypocoristique \\
\hline Hugo & Hugo l'escarbeau & Hugo l'escargot + le beau (mot-valise) \\
\hline Maëlle (Metayer) & $\begin{array}{l}\text { Elle m'a piqué mes } \\
\text { tailleurs chanel }\end{array}$ & $\begin{array}{l}\text { Verlan syllabique + paronymie }+ \\
\text { extension }\end{array}$ \\
\hline Romane & Romanoranger & Mot-valise \\
\hline Théo (Rossignol) & Rossignol puis Théolait & $\begin{array}{l}\text { Homophonie } \\
\text { «thé au lait» (calembour) }\end{array}$ \\
\hline Victor (Brosse) & Boss & Paronymie + emprunt \\
\hline
\end{tabular}

\footnotetext{
${ }^{22}$ Séance observée et filmée le 13/11/2008 en classe de CM2 (école Malherbe, Grenoble)
} 


\section{Document 4 : Production écrite de deux élèves de CLIN "Normat"}

Prise de notes

lors de l'écoute

d'un texte de slam

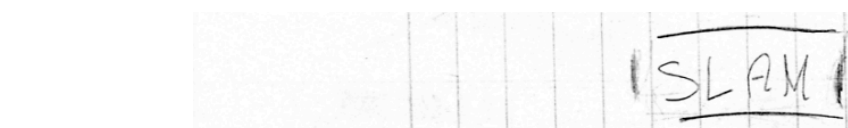

Début du texte

coproduit par

deux élèves

(premier jet)

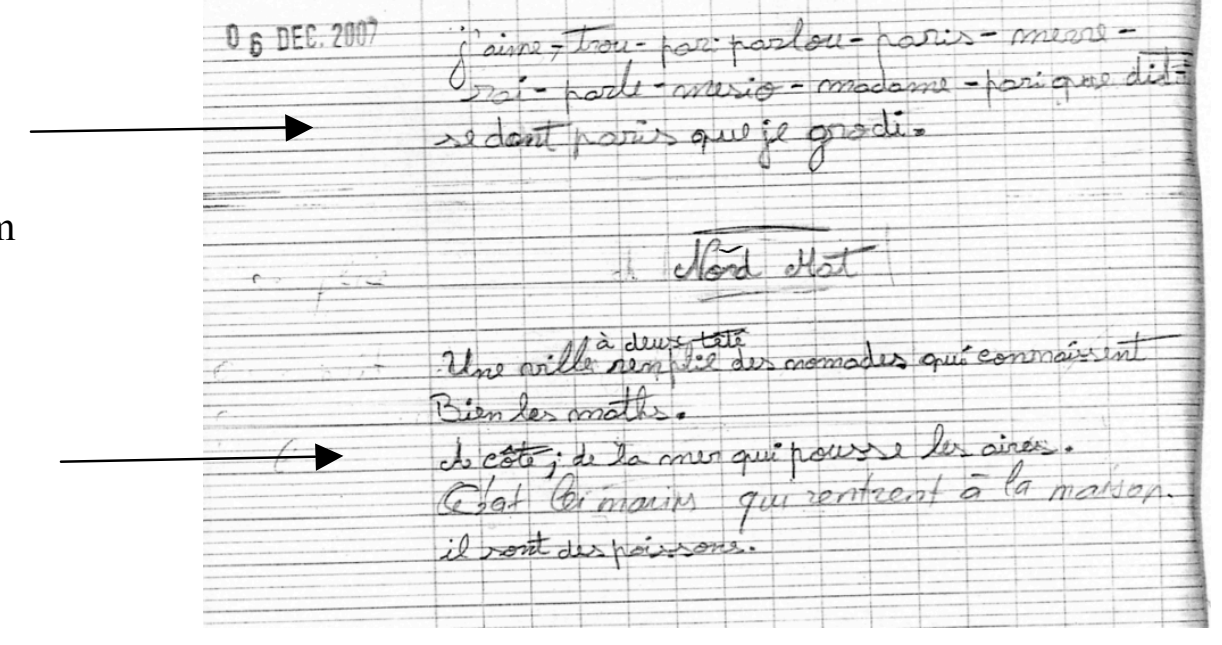

\section{Document 5 : Transcription de l'échange entre les deux élèves}

Date et lieu de l'enregistrement : le 6/12/07 à l'école Malherbe (Grenoble).

Les interlocuteurs sont désignés par les initiales de leurs prénoms.

Les conventions suivantes ont été adoptées pour la transcription :

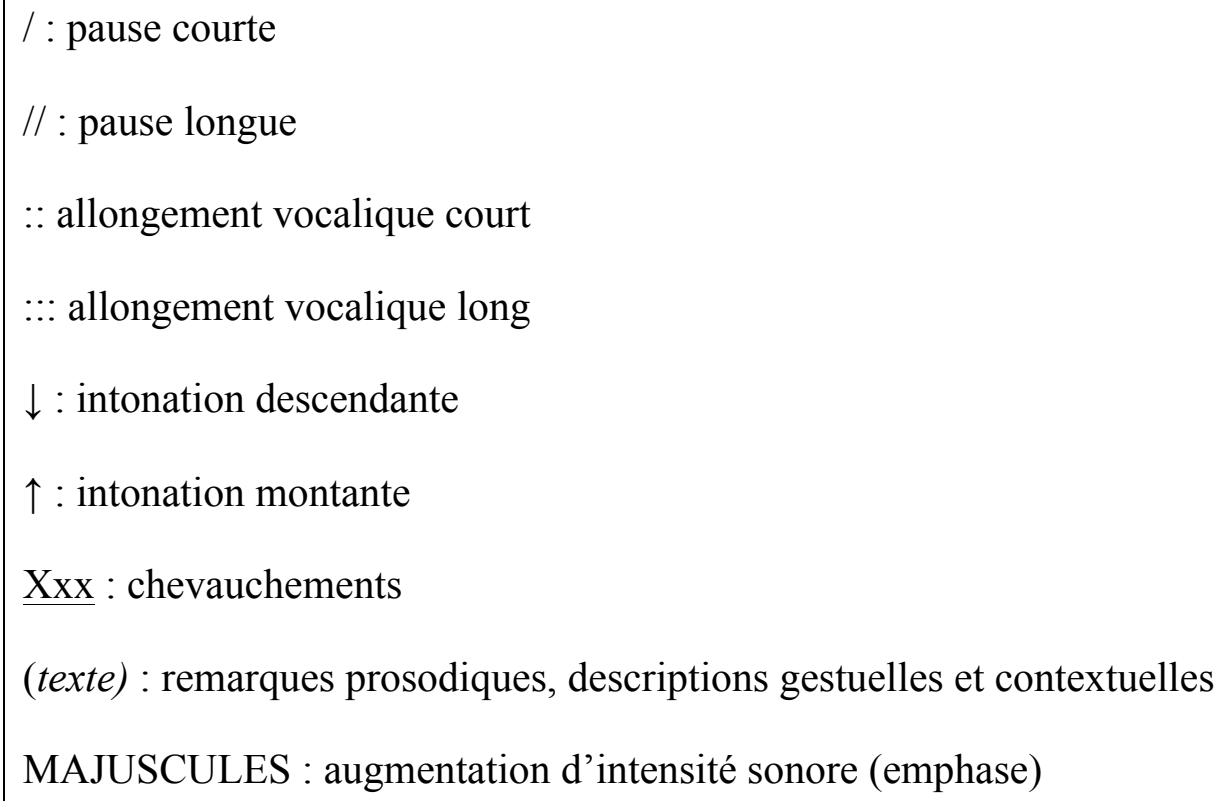


$\mathrm{N}$ : Maintenant on va commencer les rimes

M : Non : Faut mettre le paysage $\uparrow$

$\mathrm{N}$ : Moi j'm'en fiche / moi je commence les rimes $\downarrow$

$\mathrm{M}:$ Le paysage / on avait dit $\uparrow$

$\mathrm{N}$ : Oui mais le paysage moi je veux un paysage qui rime $\downarrow$

M : Á la fin / alors à la fin on mettra le paysage $\downarrow$ à la fin / non à la fin il va pas rimer / à la fin on va dire / non là on écrit :

$\mathrm{N}$ : J'avais une idée / j'avais une idée SUPER au bout de la langue mais / à cause de la salive elle est tombée / et maintenant j'ai oublié l'idée / j'avais une idée EXTRAORDINAIRE (mouvements enthousiastes des mains) / laisse-moi réfléchir (fait semblant de se boucher les oreilles) laisse moi réfléchir: ah ouais $\uparrow$ j'ai retrouvé le rythme du paysage / regarde / une : une / une / attends j'te dis mes deux phrases $\downarrow$ une ville remplie de Nomat / on connaît bien les maths // la mer : soufflée par les airs :: (mouvement des mains) ça va $\uparrow$

M (en train d'écrire): Pour l'instant oui $\downarrow$

$\mathrm{N}: \mathrm{O}$ dit ::

M : Non : mais là on écrit : euh : une ville de nomat Au COTE de la mer $\downarrow$ écris / une ville remplie de nomat au côté de la mer $\downarrow$ et après on commence les rimes / on met un point et on commence les rimes $\downarrow$

$\mathrm{N}$ : Non maintenant on commence pas ::

$\mathrm{M}$ : Ben moi j’vais faire comme ça hein

$\mathrm{N}$ : Non / après on met un point-virgule / la mer pousse les airs $\downarrow$

M : Alors maintenant t'attends un peu / j'arrive $\downarrow$ et t'as : c'est pas ON c'est QUI / qui connaît bien les maths $\downarrow$

$\mathrm{N}$ (regarde la caméra puis chuchote quelque chose à $M$, regarde à nouveau la caméra puis se cache vraisemblablement gêné par l'enregistrement) 


\section{À propos de l'auteure}

Camille Vorger est docteure en Sciences du langage à l'université Stendhal, Grenoble 3 (LIDILEM), formatrice en Lettres (IUFM de Grenoble) et en FLES (CASNAV, CUEF). Spécialisée en didactique et linguistique, elle vient de soutenir une thèse intitulée « Poétique du slam, de la scène à l'école » et sous-titrée « Néologie, néostyles et créativité lexicale », sous la direction de F. Grossmann et la co-direction de D. Abry. Ex-enseignante de CLIN, elle est aussi l'auteure d'ouvrages et guides pédagogiques en Français Langue Étrangère (sur la chanson notamment) et Seconde (CD Rom) comme en Français Langue Maternelle.

Courriel : camille.vorger@wanadoo.fr

Adresse : LIDILEM, Université Stendhal, 1180 avenue centrale, BP 25, 38040 GRENOBLE CEDEX 9, Saint Martin d'Hères, France. 